\title{
Effect of Growth and Maturation in a Hypoxic Environment on Maximum Coronary Flow Rates of Isolated Rabbit Hearts
}

\author{
GERARD HOLMES AND MICHAEL L. EPSTEIN \\ Pediatric Cardiology Research Lab, The University of Florida College of Medicine, Gainesville, Florida 32610
}

\begin{abstract}
This study was designed to determine the effect of growth and maturation of rabbits in a hypoxic $(\mathbf{H})$ environment on the ability to perfuse myocardium. Rabbits were raised in either a normoxic $(\mathrm{N})\left(\mathrm{Po}_{2}=19.7 \mathrm{kPa}\right.$ or 149 torr $)$ or an $\mathrm{H}$ environment $\left(\mathrm{PO}_{2}=8.6 \mathrm{kPa}\right.$ or $65 \pm 3$ $\mathrm{mm} \mathrm{Hg}$ ) and were studied at ages $5 \mathrm{wk}$ (immature) and 12 wk (mature). Coronary flow (CF) was measured at baseline, during infusion of a maximally dilating concentration of adenosine (A), and at peak reactive hyperemic response to transient inflow occlusion in an isolated, non-blood-perfused heart preparation. Hearts were unloaded, paced at a constant rate ( 200 beats/min), and perfused at a constant inflow pressure $(50 \mathrm{~mm} \mathrm{Hg})$. Regional $\mathrm{CF}$ was measured, using microspheres, in right ventricular, left ventricular, and atrial cardiac segments during vasodilation with $\mathrm{A}$. $\mathrm{H}$ rabbits developed right ventricular but not left ventricular segment hypertrophy/hyperplasia. CF was greater in immature than mature hearts and higher in $\mathrm{H}$ than $\mathrm{N}$ hearts during baseline, vasodilation with $A$, and at peak reactive hyperemia (two-way analysis of variance, $p \leq 0.05$ ). Regional $C F$ during vasodilation with $A$ was greater in immature than mature hearts, in both right and left ventricular segments, and higher in $\mathbf{H}$ than $\mathbf{N}$ hearts in all cardiac segments (two-way analysis of variance, $p \leq 0.05$ ). Differences in total and regional $\mathrm{CF}$ between $\mathrm{H}$ and $\mathrm{N}$ hearts were present at both study ages ( $t$ test, $p \leq 0.05$ ). The differences in $\mathrm{CF}$ at baseline show that, under conditions of reduced $\mathrm{O}_{2}$ availability (bloodless perfusate), myocardial perfusion was better in immature than mature hearts and was improved by growth in hypoxia. On the other hand, the differences in $C F$ during infusion of $A$ and at peak reactive hyperemia show that the maximum capacity for myocardial perfusion was also better in the immature hearts and further improved by growth in hypoxia, even in segments undergoing hypertrophy/hyperplasia. These findings suggest an important adaptation to environmental hypoxia in growing, maturing rabbits: an increased intrinsic vascular capacity to perfuse hypertrophied and nonhypertrophied myocardium. (Pediatr Res 33: 527-532, 1993)
\end{abstract}

Abbreviations

ANOVA, analysis of variance

Received January 22, 1992; accepted January 12, 1993

Correspondence: Gerard Holmes, M.D., Ph.D., Division of Cardiology, Department of Pediatrics, University of Florida College of Medicine, Box 100296, Gainesville, FL 32610

Supported by an American Heart Association-Florida Affiliate Clinician Scientist Award (CS89/1) (G.H.)
Acclimation of immature rodents to environmental hypoxia is associated with development of increased capillary density and capillary to myofiber ratios in both hypertrophied right and nonhypertrophied left ventricular myocardium (1-3). As a result of these increases in myocardial vascularity, intramyocardial diffusion distance for $\mathrm{O}_{2}$ is believed to be reduced, and ability to oxygenate myocardium is thought to be improved to some extent $(2,3)$. There is, however, a current paucity of data regarding the potential consequences of hypoxia-related changes in myocardial vascularity on myocardial perfusion.

Turek et al. (4) studied the effect of raising rats at simulated altitude of $3500 \mathrm{~m}$ (hypobaric hypoxia) on regional coronary flow responses to ventilation with a gas mixture containing $11 \%$ $\mathrm{O}_{2}$ (acute hypoxia). They found that coronary blood flow was greater (per g tissue weight) in hypoxically than normoxically raised rats, in both hypertrophied right and nonhypertrophied left ventricular myocardium. However, because it was not known if maximal coronary vasodilation was achieved in response to hypoxic ventilation, it cannot be determined whether the greater coronary flow values found in the hypoxically raised rats were the result of increases in myocardial vascularity or some other cause.

Manohar et al. $(5,6)$ have studied the effects of raising calves at a similar simulated altitude on regional coronary reserve. In contrast to the results of Turek et al. (4), they found that minimal coronary resistance during maximal pharmacologic coronary vasodilation with adenosine did not differ between calves raised at this simulated altitude and calves raised at sea level in any cardiac region. Thus, they concluded that chronic exposure of calves to this level of hypobaric hypoxia had no effect on the vascular capacity to perfuse myocardium. These findings in calves, however, may not be reliably extrapolated to rodent species, because species differences apparently exist in adaptation to hypoxia (7), and because rodents have been otherwise shown to have a comparatively greater capacity for myocardial vascular growth (8).

A method for raising rabbits in a hypoxic environment, beginning in the neonatal period, has been established at the University of Florida and used in our laboratory as an experimental model for studying the effects of hypoxemia on the maturing cardiovascular system $(9,10)$. The purpose of this study was to determine the effects of growth and maturation of rabbits in this hypoxic environment on the ability to perfuse myocardium.

Measurement of maximal coronary dilator responses to such pharmacologic agents as adenosine or to transient coronary inflow occlusion is considered an effective means for assessment of maximal myocardial blood flow rates and the computation of minimal resistances (11-13). Accordingly, in this study, maximal coronary flow rates were assessed during infusion of a maximally dilating concentration of adenosine and after a duration of inflow occlusion known to produce a maximal hyperemic response. Because variations in cardiac load and wall stress, coronary perfusion pressure, and heart rate (11-13) can modulate maximal coronary flow rates, experiments were performed in an isolated 
heart preparation in which these factors were controlled. This preparation also offers the advantage of eliminating potentially confounding neurohumoral influences.

\section{MATERIALS AND METHODS}

The handling and use of rabbits for this study was approved by the Committee on Animal Use at the University of Florida and was done in accordance with The Guiding Principles in Care and Use of Animals approved by the Council of the American Physiological Society. The method used for raising rabbits in a hypoxic environment has been described in detail (9). In brief, 2-d-old New Zealand White rabbits were placed in a special chamber designed to maintain an ambient $\mathrm{PO}_{2}$ of $8.6 \pm 0.4 \mathrm{kPa}$ $(65 \pm 3 \mathrm{~mm} \mathrm{Hg}$ ), at normal barometric pressure (normobaric hypoxia). Within the chamber, expired $\mathrm{CO}_{2}$ was absorbed by indicating Baralyme (Chemtron Medical Division of Allied Healthcare, St. Louis, MO), and water vapor was absorbed by indicating Drierite (WA Hammond, Xenia, OH). With the known inspired $\mathrm{O}_{2}$ concentration, the predicted arterial Po2 would be no greater than $5.3 \mathrm{kPa}(40 \mathrm{~mm} \mathrm{Hg})(9)$. The rabbits were removed from the chamber for $1 \mathrm{~h}$ each day so that they could nurse (before weaning) and the chamber could be cleaned. Normoxic rabbits were raised in an otherwise similar environment, except that ambient $\mathrm{PO}_{2}$ was $19.7 \mathrm{kPa}(149 \mathrm{~mm} \mathrm{Hg})$.

Rabbits were removed from the chambers for study at ages 5 wk (immature) or $12 \mathrm{wk}$ (mature). Each animal was anesthetized (sodium pentobarbital $172 \mu \mathrm{M} / \mathrm{kg}$ ) and heparinized (100 IU $/ \mathrm{kg}$ ) intraperitoneally. Venous blood samples were obtained for measurement of hematocrit. Rapid thoracotomy and heart excision were performed and the aortic root was cannulated to allow coronary perfusion. After onset of perfusion, the left ventricle was vented to atmospheric pressure through an apical stab incision; thus, the left ventricle was unloaded. Similarly, the pulmonary artery was opened to atmospheric pressure, unloading the right ventricle. Hearts were paced via the right ventricle at 200 beats/min. Coronary perfusion pressure was maintained at $50 \mathrm{~mm} \mathrm{Hg}$, except during periods of total inflow occlusion.

Total coronary flow was measured by a cannulating (2-mm internal diameter) Biotronex electromagnetic flowmeter. Coronary inflow pressure was measured by a Statham P23 ID transducer (Gould Inc., Cleveland, $\mathrm{OH}$ ). Surface electrograms were monitored using left atrial and left ventricular electrodes. Pressure, flow, and electrocardiographic signals were recorded with a Honeywell 1858 CRT Visicorder (Honeywell Inc., Medical Electronics Div., Pleasantville, NY).

The perfusion fluid was modified Krebs-Henseleit solution containing: $\mathrm{NaCl} 122 \mathrm{mM}, \mathrm{KCl} 4.7 \mathrm{mM}, \mathrm{MgSO}_{4} 1.2 \mathrm{mM}, \mathrm{CaCl}_{2}$ $2.5 \mathrm{mM}, \mathrm{NaHCO}_{3} 22 \mathrm{mM}$, and glucose $8 \mathrm{mM}$. It was oxygenated with a gas mixture of $95 \% \mathrm{O}_{2}, 5 \% \mathrm{CO}_{2}$ to produce a $\mathrm{PO}_{2}>66.3$ $\mathrm{kPa}(500 \mathrm{~mm} \mathrm{Hg}), \mathrm{pH} 7.35 \pm 0.05$, and $\mathrm{PCO}_{2}=5.4 \pm 0.7 \mathrm{kPa}$ $(40 \pm 5 \mathrm{~mm} \mathrm{Hg}$ ), as determined by measurement of perfusion fluid samples using a Corning $168 \mathrm{pH} /$ blood gas analyzer (Corning Medical, Medfield, MA). Temperature of the perfusion fluid and the heart was maintained at $37^{\circ} \mathrm{C}$ by a circulating water bath and heat exchanger.

Experimental protocols. Experimental protocols were initiated after a 30 -min equilibration period during which coronary flow stabilized. In a first set of experiments, isolated hearts of 5-wkold rabbits ( $n=12$ normoxic, $n=13$ hypoxic) were exposed to $10 \mu \mathrm{M}$ adenosine in the perfusate, and coronary flow was measured at steady state responses. This concentration of adenosine is several times greater than that previously found to induce maximum coronary vasodilator response in isolated hearts from rabbits of similar maturational stages (14).

In a second set of experiments in hearts of 5-wk-old rabbits ( $n$ $=13$ normoxic, $n=12$ hypoxic), maximal coronary flow response to $90 \mathrm{~s}$ of total coronary inflow occlusion was measured. This duration of inflow occlusion was previously shown to induce maximal response in isolated hearts of rabbits of similar maturational stages (14).

A third series of experiments was performed in hearts of 12wk-old rabbits ( $n=12$ normoxic, $n=13$ hypoxic). In this series, maximal responses to $90-\mathrm{s}$ inflow occlusion were first determined, and, after return of coronary flow to baseline, response to perfusion with Krebs-Henseleit solution containing $10 \mu \mathrm{M}$ adenosine was measured.

Segmental flow at maximum dilation with adenosine. Regional flow distribution at maximal coronary dilation with adenosine was measured using radiolabeled microspheres. At steady state vasodilator response to adenosine, $0.1 \mathrm{~mL}(5 \mathrm{wk})$ or $0.2 \mathrm{~mL}(12$ wk) of a well agitated, premixed solution of normal saline $(0.9 \%)$ containing approximately $1000000 \mathrm{I}^{125}$-labeled, $15-\mu \mathrm{m}$ microspheres/mL and 1\% Tween 80 (Medical-Surgical Division/3M Company, St. Paul, MN) was injected into a stream of perfusion fluid that coursed through the center of a bubble trap located in line with the perfusion apparatus (Fig. 1). This bubble trap added compliance to the apparatus and allowed injection of microspheres without appreciable alteration of coronary flow or perfusion pressure. Moreover, the impact of the stream on the distal perfusate column caused turbulence, promoting mixture of the microspheres with the perfusion fluid.

Three min were alloted for injected microspheres to label the heart. Total elapsed time for each experiment was less than 90 min. Experiments were then terminated. To determine weight and radioactivity, the hearts were divided into three segments: 1) the atria; 2) the left ventricular free wall; and 3) the right ventricular free wall plus the ventricular septum. Hearts were

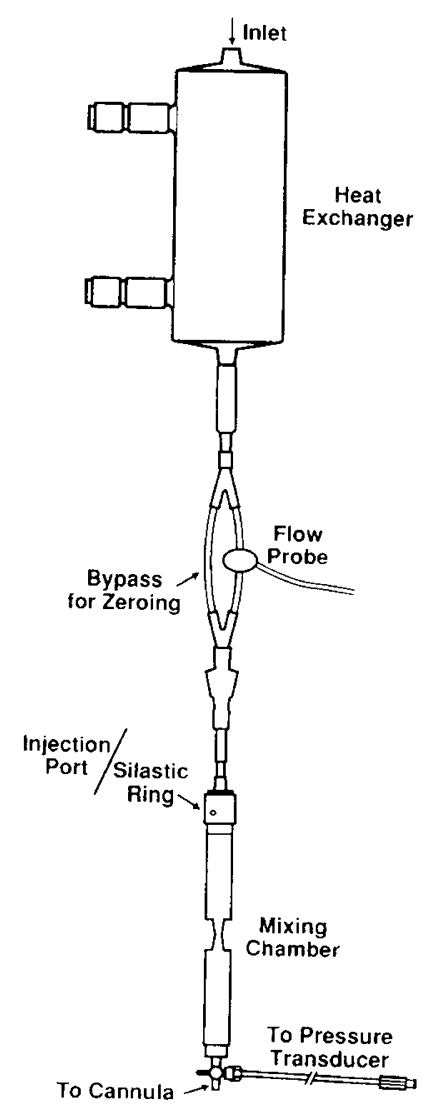

Fig. 1. Schematic of the modified Langendorf perfusion apparatus used in this study. Premixed microspheres were injected into a bubble trap through a port covered by an air-tight ring of Silastic tubing. Perfusate coursed through the center of the trap. The bubble trap added compliance to the system and allowed injection of microspheres without alteration of coronary flow or inflow pressure. Turbulence occurred in the perfusate column distal to the bubble trap, promoting mixture of microspheres with the perfusion fluid. 
divided in this manner so that flow response in segments not undergoing hypertrophy could be compared with that in segments undergoing hypertrophy. For purpose of discussion, the cardiac segment consisting of the right ventricular free wall and the ventricular septum will be referred to as the right ventricular segment.

Radioactivity of all samples was counted in a gamma counter (Beckman 5500), with all tissue fragments less than $1.0 \mathrm{~cm}$ from the bottom of the vial. Fractional activity of each segment was calculated as: segmental fractional activity = segmental activity/ total heart activity. Segmental flow was calculated as: segmental flow $=$ total flow times segmental fractional activity.

The radioactivity of similarly treated $0.1-\mathrm{mL}$ samples of the microsphere solution was measured directly and served as a reference value for calculation of the total number of microspheres labeling each heart and each cardiac segment, using the following equations: Microspheres in each heart $=100000$ times heart activity/reference activity. Microspheres in each segment $=$ segmental fractional activity times microspheres in heart.

Statistical analysis. Data are expressed as mean \pm SEM. Statistical comparisons to determine differences in total values during baseline, maximum vasodilation with adenosine, and at peak reactive hyperemia were made using one-way ANOVA and Scheffe's test. Similarly, one-way ANOVA and Scheffe's test were done to determine statistical differences among regional coronary flow values at maximal vasodilation with adenosine. Statistical comparisons to determine effects of maturation (immature and mature) and environmental $\mathrm{O}_{2}$ level (hypoxic and normoxic) were made using a two-way ANOVA. Statistical comparisons to determine effects of environmental $\mathrm{O}_{2}$ level at the same maturational stage (study age) were made using $t$ test. A $p$ value $\leq$ 0.05 was considered significant for ANOVA, Scheffe's, and $t$ tests. Variabilities in total and segmental coronary flow during maximal vasodilation with adenosine were compared using the Morgan-Pittman test. A $p$ value $\leq 0.008$ was considered statistically significant for these comparisons.

\section{RESULTS}

Hypoxically raised rabbits had no sign of congestive heart failure or dehydration but did have higher hematocrit values than the normoxically raised controls (Table 1). Although body weight, total heart weight, and left ventricular segment weight were similar in hypoxically and normoxically raised rabbits at age $5 \mathrm{wk}$, they were lower in the hypoxically raised group at age 12 wk. In contrast, right ventricular segment weight was higher in the hypoxic group at age $5 \mathrm{wk}$ but was similar to that of the normoxic group at age $12 \mathrm{wk}$. Atrial segment weights did not differ between hypoxic and normoxic groups at either study age.

To help assess differences in cardiac segment size in the face of differences in body weight, cardiac segment:body weight ratios and right ventricular:left ventricular segment ratios were also compared (Table 2). All cardiac segment:body weight ratios were higher in immature (5 wk) than in mature (12 wk) rabbits. Right ventricular segment:body weight ratios were $28 \%$ higher at age 5 wk and $18 \%$ higher at age $12 \mathrm{wk}$ in hypoxically compared with normoxically raised rabbits. Right ventricular:left ventricular weight ratios were also higher in hypoxically than normoxically raised rabbits at both study ages. Similar results were found for atrial:body weight ratios. Left ventricular segment:body weight ratios, however, did not differ between these groups at either study age. These results show that the relative masses of the right ventricular and atrial segments were increased, whereas the relative mass of the left ventricular segment was unchanged in the hypoxically raised rabbits.

Coronary flow. Total coronary flow (Fig. 2) differed under the three experimental conditions: baseline, maximal vasodilation with adenosine, and peak reactive/hyperemic response to inflow occlusion ( $p \leq 0.001$, one-way ANOVA). Peak reactive hyperemic responses were greater than adenosine responses, and both peak reactive hyperemic and adenosine responses exceeded baseline $(p \leq 0.001)$.

Baseline coronary flow. Values for baseline coronary flow $(\mathrm{mL} /$ $\mathrm{min} / \mathrm{g}$ ) were: $5.7 \pm 0.3$ (5-wk normoxic), $6.8 \pm 0.6$ (5-wk hypoxic), $4.6 \pm 0.6$ (12-wk normoxic), and $5.9 \pm 0.4$ (12-wk hypoxic). Baseline flow was, in general, greater in immature than mature hearts, and higher in hypoxic than normoxic hearts. Specifically, baseline flow rates were $19 \%$ higher at age $5 \mathrm{wk}$ and $28 \%$ higher at age $12 \mathrm{wk}$ in hearts of hypoxically raised compared with normoxically raised rabbits.

Adenosine. Similar percentages of the microspheres injected during maximal coronary vasodilation with adenosine were found to label hearts in all experimental groups (group ranges 64 $\pm 7 \%$ to $75 \pm 10 \%$ ). All cardiac segments were found to contain more than 4000 microspheres.

Values for total and segmental coronary flow $(\mathrm{mL} / \mathrm{min} / \mathrm{g}$ ) during infusion of adenosine are given in Table 3 . Total coronary flow during infusion of adenosine (Fig. 2) was, in general, greater in immature than mature hearts and higher in hypoxically raised than in normoxically raised rabbits. Specifically, total coronary flow rates during adenosine infusion were $30 \%$ higher at age 5 wk and $47 \%$ higher at age $12 \mathrm{wk}$ in hearts of hypoxically raised compared with normoxically raised rabbits.

Coronary flow during vasodilation with adenosine was similar in right ventricular and left ventricular segments but was greater in both of these segments than in the atrial segment $(p \leq 0.001$, Scheffe's test). Segmental coronary flow values were greater in immature than mature hearts in both ventricular segments and higher in hypoxically raised than in normoxically raised rabbits in all cardiac segments. Right ventricular, left ventricular, and atrial segment flow values were, respectively, 21,30 , and $51 \%$ higher at age $5 \mathrm{wk}$ and 48,32, and $64 \%$ higher at age $12 \mathrm{wk}$ in hearts of hypoxically raised compared with normoxically raised rabbits.

The variabilities in segmental coronary flow measurements (microspheres) were similar to those of total coronary flow measurements (electromagnetic flow probe) for the right and left ventricular segments but were lower than that of total flow for the atrial segments (Morgan-Pittman test).

Inflow occlusion. Values for peak reactive hyperemic flow responses to transient coronary inflow occlusion were $(\mathrm{mL} / \mathrm{min} /$ g): $12.2 \pm 1.2$ (5-wk normoxic); $15.2 \pm 1.1$ (5-wk hypoxic); 8.2

Table 1. Hematocrits, body weights, and cardiac segment weights*

\begin{tabular}{ccccccccc}
\hline Age (wk) & Group & $n$ & \multicolumn{1}{c}{ Hct $(\%)$} & \multicolumn{1}{c}{ Body wt $(\mathrm{g})$} & \multicolumn{1}{c}{ Heart wt $(\mathrm{g})$} & \multicolumn{1}{c}{ RV wt $(\mathrm{g})$} & \multicolumn{1}{c}{ LV wt $(\mathrm{g})$} & AT wt $(\mathrm{g})$ \\
\hline 5 & $\mathrm{~N}$ & 25 & $0.36 \pm 0.01$ & $747 \pm 30$ & $2.4289 \pm 0.1061$ & $1.1766 \pm 0.0506$ & $0.7516 \pm 0.0401$ & $0.5007 \pm 0.0300$ \\
5 & $\mathrm{H}$ & 25 & $0.42 \pm 0.02 \dagger \ddagger$ & $667 \pm 43 \dagger$ & $2.5139 \pm 0.1182 \dagger$ & $1.2743 \pm 0.0518 \ddagger$ & $0.7269 \pm 0.0291 \dagger$ & $0.5127 \pm 0.0343$ \\
12 & $\mathrm{~N}$ & 12 & $0.37 \pm 0.01$ & $2299 \pm 77 \S$ & $5.8621 \pm 0.2502 \S$ & $2.8857 \pm 0.1186 \S$ & $1.9638 \pm 0.1311 \S$ & $1.0126 \pm 0.0719 \S$ \\
12 & $\mathrm{H}$ & 13 & $0.44 \pm 0.01 \dagger \|$ & $1783 \pm 113 \uparrow \S \|$ & $4.9604 \pm 0.3845 \dagger \S \|$ & $2.6760 \pm 0.2399 \S$ & $1.3817 \pm 0.1311 \uparrow \S$ & $0.9032 \pm 0.1006 \S$ \\
\hline
\end{tabular}

* Values are expressed as mean $\pm \mathrm{SEM} ; \mathrm{N}$, raised in normoxia; $\mathrm{H}$, raised in hypoxia; $\mathrm{RV}$, right ventricular segment; LV, left ventricular segment; $\mathrm{AT}$, atrial segment; Hct, hematocrit. $5 \mathrm{wk}=$ immature; $12 \mathrm{wk}=$ mature.

$\dagger \mathrm{H}$ differs from $\mathrm{N}$ (two-way ANOVA, $p \leq 0.05$ ).

$\ddagger$ Differs from 5 -wk N $(t$ test, $p \leq 0.05)$.

$\S$ Twelve-wk differ from 5-wk (two-way ANOVA, $p \leq 0.05$ ).

\| Differs from 12-wk N $(t$ test, $p<0.05)$. 
Table 2. Cardiac segment:body weight ratios*

\begin{tabular}{ccccccc}
\hline Age $(w k)$ & Group & $n$ & $\begin{array}{c}\text { RV:body wt } \\
\left(\times 10^{-4}\right)\end{array}$ & $\begin{array}{c}\text { LV:body wt } \\
\left(\times 10^{-4}\right)\end{array}$ & $\begin{array}{c}\text { AT:body wt } \\
\left(\times 10^{-4}\right)\end{array}$ & RV:LV wt \\
\hline 5 & $\mathrm{~N}$ & 25 & $15.9 \pm 0.5$ & $10.1 \pm 0.4$ & $6.7 \pm 0.3$ & $1.64 \pm 0.11$ \\
12 & $\mathrm{H}$ & 25 & $20.4 \pm 1.3 \dagger \ddagger$ & $11.7 \pm 0.7$ & $8.0 \pm 0.4 \pm \S$ & $1.75 \pm 0.06 \dagger$ \\
12 & $\mathrm{~N}$ & 12 & $12.6 \pm 0.4 \S$ & $8.6 \pm 0.6 \S$ & $4.4 \pm 0.3 \S$ & $1.58 \pm 0.18$ \\
\hline
\end{tabular}

* Values are expressed as mean \pm SEM. $5 \mathrm{wk}=$ immature; $12 \mathrm{wk}=$ mature. Abbreviations are the same as in Table 1.

$\dagger \mathrm{H}$ differs from $\mathrm{N}$ (two-way ANOVA, $p \leq 0.05$ ).

$\ddagger$ Differs from 5-wk $N(t$ test, $p \leq 0.05)$.

$\S$ Twelve-wk differ from 5-wk (two-way ANOVA, $p \leq 0.05$ ).

$\|$ Differs from 12-wk N $(t$ test, $p \leq 0.05)$.

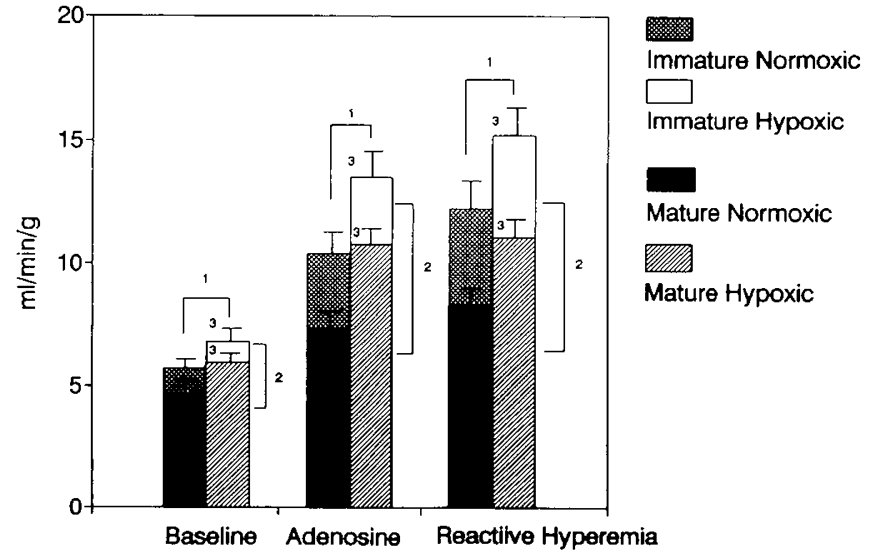

Fig. 2. Total coronary flow $(\mathrm{mL} / \mathrm{min} / \mathrm{g})$ in isolated rabbit hearts during baseline, adenosine infusion, and at peak reactive hyperemia. 1 , Hypoxic differ from normoxic (two-way ANOVA, $p \leq 0.05$ ); 2 , mature (12-wk) differ from immature (5-wk) (two-way ANOVA, $p \leq 0.05$ ); 3 , differs from age-matched normoxic group ( $t$ test, $p \leq 0.05$ ). Total coronary flow was greater in immature (5-wk) than mature (12-wk) hearts and higher in hearts of rabbits raised in hypoxia than normoxia under all three experimental conditions. Differences between hypoxically and normoxically raised rabbits were present at both maturational stages.

\pm 0.6 (12-wk normoxic), and $11.0 \pm 0.8$ (12-wk hypoxic). Peak reactive hyperemic responses were greater in immature than in mature hearts and higher in hearts of hypoxically raised than normoxically raised rabbits. Peak reactive hyperemic flow rates were $25 \%$ higher at age $5 \mathrm{wk}$ and $34 \%$ higher at age $12 \mathrm{wk}$ in hearts of hypoxically raised compared with normoxically raised rabbits.

\section{DISCUSSION}

Previous studies in which the present experimental method for raising rabbits in environmental hypoxia was used have demonstrated increased right but not left ventricular weight and protein content by age $3 \mathrm{wk}(9,10)$. Consistent with these findings, the present results show increased right ventricular segment weight but unchanged left ventricular segment weight in hypoxically raised rabbits at age $5 \mathrm{wk}$. Taken together, these findings demonstrate that right but not left ventricular hypertrophy/hyperplasia developed as consequence of exposure of maturing rabbits to the level of hypoxia used.

In contrast to these findings at ages 3 and $5 \mathrm{wk}$, hypoxically raised rabbits had right ventricular segment weights similar to those of normoxically raised controls at age $12 \mathrm{wk}$. This lack of increased right ventricular segment weight can be attributed to a generalized growth failure because body weight, heart weight, and left ventricular segment weight were lower in the hypoxically raised group at this age (Table 1). It is noteworthy that this hypoxia-related growth failure could not have been caused by inadequate maternal lactation or opportunity to nurse, because it was manifested after age $5 \mathrm{wk}$, an age when rabbits feed independently.

Effects of maturation in hypoxia on ability to perfuse myocardium baseline coronary flow. In this study, hearts were perfused with a physiologic buffer solution that lacked $\mathrm{Hb}\left(\mathrm{O}_{2}\right.$ content approximately $0.157 \mathrm{~mL} \mathrm{O}_{2} / \mathrm{dL}$ ). Thus, the observed differences in baseline flow values (Fig. 2) among immature and mature hearts and in hearts of hypoxically and normoxically raised rabbits show that, under conditions of similarly reduced arterial $\mathrm{O}_{2}$ availability, myocardial perfusion and $\mathrm{O}_{2}$ delivery were better in immature than mature hearts and were increased in response to growth in environmental hypoxia.

Because myocardial $\mathrm{O}_{2}$ consumption was not measured in this study, it cannot be determined directly whether myocardial oxygenation was also changed with maturation and exposure to hypoxia in a manner similar to myocardial perfusion and $\mathrm{O}_{2}$ delivery. In this regard, it is helpful to interpret the present findings in the light of previous findings by Matherne et al. (15) and Turek et al. (4).

Matherne et al. (15) evaluated the effects of varying perfusate oxygenation in immature ( 2 to $4 \mathrm{wk}$ ) and mature (4 to $6 \mathrm{mo}$ ) rabbit hearts. They used an isolated heart preparation similar to that of the present study with the exception that perfusion was delivered at a constant flow. Despite finding statistically similar values for myocardial $\mathrm{O}_{2}$ consumption in immature and mature hearts, coronary conductance was found to be higher in hearts of immature compared with mature rabbits during perfusion with medium containing an amount of $\mathrm{O}_{2}$ similar to that of the present study (modified Krebs-Henseleit solution equilibrated

Table 3. Total and segmental coronary flow during adenosine dilation $(\mathrm{mL} / \mathrm{min} / \mathrm{g})^{*}$

\begin{tabular}{ccccccl}
\hline Age (wk) & Group & $n$ & Total & RV & LV & AT \\
\hline 5 & $\mathrm{~N}$ & 12 & $10.4 \pm 0.9$ & $12.8 \pm 1.4$ & $11.9 \pm 1.4$ & $3.5 \pm 0.4$ \\
5 & $\mathrm{H}$ & 13 & $13.5 \pm 1.2 \dagger \ddagger$ & $15.5 \pm 1.5 \dagger \ddagger$ & $15.5 \pm 1.3 \dagger \ddagger$ & $5.3 \pm 0.7 \dagger \ddagger$ \\
12 & $\mathrm{~N}$ & 12 & $7.3 \pm 0.7 \S$ & $7.9 \pm 0.9 \S$ & $9.8 \pm 1.5 \S$ & $2.8 \pm 0.4$ \\
12 & $\mathrm{H}$ & 13 & $10.7 \pm 0.7 \dagger \S \|$ & $11.7 \pm 0.9 \dagger \S \|$ & $12.9 \pm 1.0 \dagger \S \|$ & $4.6 \pm 0.4 \dagger \|$ \\
\hline
\end{tabular}

* Values are expressed as mean $\pm \mathrm{SEM} ; 5 \mathrm{wk}=$ immature; $12 \mathrm{wk}=$ mature. Abbreviations are the same as in Table 1. Total coronary flow was measured via electromagnetic flow probe, and segmental flow was measured using microspheres.

$\dagger \mathrm{H}$ differs from $\mathrm{N}$ (two-way ANOVA, $p \leq 0.05$ ).

$\ddagger$ Differs from 5-wk N ( $t$ test, $p \leq 0.05$ ).

$\S$ Twelve-wk differ from 5-wk (two-way ANOVA, $p \leq 0.05$ ).

$\|$ Differs from 12-wk N $(t$ test, $p \leq 0.05)$. 
with $95 \% \mathrm{O}_{2}$ ). Similar differences were also found during perfusion with medium containing a lower amount of $\mathrm{O}_{2}$ (modified Krebs-Henseleit solution equilibrated with $10 \% \mathrm{O}_{2}$ ). These findings demonstrate maturational differences in the relationship between coronary conductance and myocardial $\mathrm{O}_{2}$ demand under conditions of reduced arterial $\mathrm{O}_{2}$ availability in rabbits. These differences predict improved myocardial perfusion and oxygenation in immature compared with mature rabbit hearts under circumstances, such as were used in the present study, in which coronary flow was allowed to be physiologically regulated. For this reason, we believe that myocardial oxygenation was also likely better in immature than mature rabbit hearts in the present study.

Turek et al. (4) studied the effect of chronic exposure of young rats to a simulated high altitude of $3500 \mathrm{~m}$ (hypobaric hypoxia) on coronary flow response to acute ventilation with a hypoxic gas mixture. Again, despite finding similar values for myocardial $\mathrm{O}_{2}$ consumption in hypoxically and normoxically raised rats, greater coronary flow was found in all cardiac regions of the rats raised in hypoxia. This means that, during acute reduction of arterial $\mathrm{O}_{2}$ availability, prior growth of rats in a hypoxic environment was associated with a comparative improvement in both myocardial perfusion and oxygenation. It is reasonable to assume that prior growth in environmental hypoxia would also result in a comparative improvement in myocardial oxygenation during periods of reduced arterial $\mathrm{O}_{2}$ availability in rabbits, inasmuch as they are also a rodent species and thus are likely to adapt to hypoxia in a manner similar to rats.

Maximal coronary flow rates. Several factors may have contributed to the differences in maximal coronary flow rates that were found in the present study (Fig. 2, Table 3). However, the fact that they were present during both maximal pharmacologic (adenosine) and metabolically induced (reactive hyperemia) coronary vasodilation suggests that they were the result of structural differences in the vascular bed.

One plausible explanation for the presently observed differences in maximal coronary flow rates between hypoxically and normoxically raised rabbits is altered arterial and/or arteriolar wall:lumen ratio. Increased arterial and arteriolar wall:lumen ratios have been found in chronically hypertensive animals and are thought to play a role in the decreased maximal coronary conductance that has been described in these animals (16). It is conceivable that decreased arterial diastolic pressure developed during hypoxic exposure due to increased tissue blood flow demand and resulted in morphologic changes opposite those seen in hypertensive animals.

The differences in maximal flow rates in this study parallel changes in myocardial capillarity that have previously been demonstrated in immature and mature animals (17) and in response to long-term exposure of maturing rodents to hypoxia $(1-3)$. Thus, it would seem most likely that they occurred as a consequence of differences in myocardial microvascular density and relative cross-sectional area of the myocardial microvascular bed (i.e. cross-sectional area of the microvascular bed per unit cardiac area). In this case, differences in the density and relative cross-sectional area of the precapillary resistance vessels may also be implicated, inasmuch as alteration of myocardial capillary cross-sectional area is thought to have insignificant effect on maximum coronary flow rates in the beating heart (18).

Maximal segmental flow. Several findings suggest that use of microspheres for determination of segmental distribution of coronary flow during maximal coronary vasodilation with adenosine in the present study is valid. First, in accordance with accepted use of microspheres for determination of regional flow distribution $(19,20)$, use of microspheres caused no appreciable alteration of the conditions of perfusion and more than adequately labeled the cardiac segments for statistical purposes. Second, because similar percentages of injected microspheres were found to label hearts in each experimental group, the findings regarding segmental flow distribution should have equivalent reliability in all experimental groups. Finally, as the variabilities of segmental flow values were similar or less than total flow values (MorganPittman test), use of microspheres did not introduce increased randomness into the determination of segmental flow. This latter observation suggests that either a homogeneous mixture of microspheres and perfusate was achieved proximal to coronary inflow or that sufficient numbers of microspheres were used to compensate for any inhomogeneity (20).

Our results show that maximal coronary flow rates were increased in hearts of hypoxically raised rabbits, both in the right ventricular segment, in which there was concurrent evidence of hypertrophy/hyperplasia, and in the left ventricular segment, in which there was no such evidence. Our finding in the right ventricular segment contrasts with results from most earlier studies, performed in adults, concerning the effects of cardiac hypertrophy on maximal myocardial blood flow rates $(8,11-$ 13). These studies showed maximal myocardial flow rates to be reduced by development of cardiac hypertrophy. They suggest that the capacity for myocardial vascular growth is limited in adults and is outstripped by development of most forms of myocardial hypertrophy (12). More recent developmental studies, however, have shown maximal myocardial blood flow rates to be unchanged when hypertrophy/hyperplasia develops early in life and to be increased when hypertrophy develops in utero (21). Moreover, maximal myocardial flow rates have also been found to be increased when cardiac hypertrophy is induced by thyrotoxicosis (22). Thus, it is now thought that developmental stage and nature of the hypertrophy-inducing stimulus can significantly modulate interaction between myocardial vascular growth and myocardial hypertrophy (12). Accordingly, the present finding regarding maximal flow rates in hypertrophied right ventricular segments of immature hypoxically raised rabbits may reasonably be interpreted as showing that, during early postnatal development and in the presence of hypoxemia, myocardial vascular growth is able to outstrip development of moderate myocardial hypertrophy/hyperplasia in rabbits.

Our results showing increased maximal coronary flow rates in nonhypertrophied left ventricular segments of hypoxically raised rabbits, on the other hand, have two different implications regarding myocardial microvascular growth. The fact that this response was found at age $5 \mathrm{wk}$, when left ventricular segment mass was similar in both hypoxically and normoxically raised rabbits, suggests that hypoxemia or a closely related factor stimulated microvascular growth in this segment. However, the fact that this response was present at age $12 \mathrm{wk}$, at which time generalized growth failure was manifested in the hypoxically raised rabbits, including in the left ventricular segment, suggests that there could have also been a hypoxia-related inhibition of normal maturational changes in the relationship between the myocardium and coronary vasculature. Such a maturational inhibition could have preserved the higher microvascular crosssectional area per unit cardiac area characteristic of early maturation.

The present findings concerning the effect of maturation of rabbits on maximal coronary flow rates are similar to previous findings of Toma et al. (17) in guinea pigs. These investigators found that postnatal growth and maturation were associated with a decrease in maximal coronary flow rate per unit cardiac mass during pharmacologic vasodilation of isolated hearts with adenosine and nitroprusside.

The present findings regarding the effect of growth and maturation in hypoxia on maximal myocardial flow rates conflict with those of Manohar et al. $(5,6)$. These investigators showed that long-term exposure of weanling calves to hypoxia caused no change in maximal coronary conductance in any cardiac region during infusion of a maximally dilating dose of adenosine in any cardiac region. Existing evidence $(1,11,12)$ suggests that this variance in experimental findings may have resulted from a number of causes, including: 1 ) interspecies differences in adaptive responses to hypoxia; 2 ) differences in maturational stage at 
onset of hypoxic exposure (neonate versus weanling); and 3) differences in experimental preparation (isolated, unloaded, paced, and constant pressure-perfused hearts versus intact animals). Although presently available data do not allow us to distinguish among these possibilities, we speculate that they are most likely due to species variation, because rodents have been otherwise shown to have a comparatively increased capacity for microvascular growth (8).

Finally, the present findings support and extend the previous findings of Turek et al. (4) by demonstrating improved myocardial perfusion in a rodent species both under conditions of reduced $\mathrm{O}_{2}$ availability and during maximal pharmacologic and metabolic coronary vasodilation. They suggest a potentially important adaptation to the level of environmental hypoxia studied in maturing rabbits: an improved vascular capacity to perfuse and oxygenate both hypertrophied and nonhypertrophied myocardium.

\section{REFERENCES}

1. Hudlicha O 1979 Capillary growth and adaptation. In: Berne RM (ed) Handbook of Physiology Society, Sect 2, Vol IV, Pt 1. American Physiological Society, Bethesda, MD, pp 165-216

2. Kaynor SR, Banchero N 1985 Myocardial capillarity in acclimation to hypoxia. Pflugers Arch 404:319-325

3. Turek Z, Grandtner M, Kreutzer F 1972 Cardiac hypertrophy, capillary and muscle fiber density, muscle fiber diameter, capillary radius and diffusion distance in the myocardium of rats adapted to an altitude of $3,500 \mathrm{~m}$. Pflugers Arch 335:19-28

4. Turek Z, Turek-Maischeider M, Classens RA, Rignalda BEM, Kreutzer F 1975 Coronary blood flow in rats native to simulated high altitude and in rats exposed to it later in life. Pflugers Arch 335:49-62

5. Manohar M, Parks CR, Busch MA, Tranquelli WJ, Bisgard GE 1982 Regional myocardial blood flow and coronary vascular reserve in unanesthetized young calves exposed to a simulated high altitude or $3500 \mathrm{~m}$ for 8 to 10 weeks. Circ Res 50:714-726

6. Manohar M, Parks CM, Busch MA, Bisgard GE 1985 Transmural coronary vasodilator reserve and flow distribution in unanesthetized calves sojourning at $3500 \mathrm{~m}$. J Surg Res 39:499-509

7. Weil JV 1990 Lessons from high altitude. Chest 97(SI):705-765

8. Scheurer J, Buttrick P 1987 The cardiac hypertrophic responses to pathologic and physiologic loads. Circulation 75(SI):63-68

9. Prestwich KN, Buss DD, Posner P 1984 A new method for raising neonatal rabbits in an hypoxic environment. J Appl Physiol 57:1913-1916

10. Posner P, Baker SP, Epstein ML, MacIntosh BK, Buss DD 1991 Effects of chronic hypoxia during maturation on the negative chronotropic effects of $\left(\mathrm{H}^{+}\right)$in the rabbit sino-atrial node. Biol Neonate 59:109-113

11. Bache R 1988 Effect of hypertrophy on the coronary circulation. Prog Cardiovasc Dis 31:403-440

12. Marcus ML, Harrison DG, Chilean WM, Koyangi S, Inou T, Tomanek RJ, Martins JB, Eastin BA, Hiratzka LF 1987 Alterations in the coronary circulation in hypertrophied ventricles. Circulation 75(SI):1-19

13. Hoffman JIE 1987 A critical review of coronary reserve. Circulation 75(SI):16

14. Buss DD, Hennemann WW, Posner P 1987 Maturation of coronary responsiveness to adenosine in the rabbit. Basic Res Cardiol 82:290-296

15. Matherne GP, Headrick JP, Coleman SD, Berne RM 1990 Interstitial transudate purines in normoxic and hypoxic immature and mature rabbit hearts. Pediatr Res 28:348-353

16. Tomanek RJ 1990 Response of the coronary vasculature to myocardial hypertrophy. JACC 15:528-533

17. Toma BS, Wangler RD, DeWitt DF, Sparks HV 1985 Effect of development on coronary vasodilator reserve in the isolated guinea pig heart. Circ Res 57:538-544

18. Chilean WM, Eastham CL, Marcus ML 1986 Microvascular distribution of coronary vascular resistance in beating left ventricle. Am J Physio 251:H779-H788

19. Buckberg GD, Luck JC, Payne B, Hoffman JIE, Archie JP, Fixler DE 1971 Some sources of error in measuring regional blood flow with radioactive microspheres. J Appl Physiol 31:598-604

20. Yipintsoi T, Dobbs WA, Scanlon PD, Knapp TJ, Bassingthwaithe JB 1975 Regional distribution of diffusable tracers and carbonized microspheres in the left ventricle of isolated dog hearts. Circ Res 33:573-587

21. Vlahakes GJ, Turley K, Verrier ED, Hoffman JIE 1980 Greater maximal coronary flow in conscious lambs with experimental right ventricular hypertrophy. Circulation 62(SII):111(abstr)

22. Chilean WM, Wangler RD, Peters KC, Marcus ML, Tomanek RJ 1985 Thyroxine-induced left ventricular hypertrophy in the rat: anatomical and physiological evidence for angiogenesis. Circ Res 57:591-598 